\title{
Pollution from Hazardous Landfill Sites
}

\author{
C. P. Please ${ }^{1} \dagger$, R. Omer $^{2}$ and G. Kirsten ${ }^{3}$ \\ ${ }^{1}$ Oxford Centre for Collaborative Applied Mathematics, University of Oxford, UK \\ 2 Arican Institute for Mathematical Sciences, Muizenberg, Cape Town, South Africa; and Department \\ of Mathematical Sciences, University of Stellenbosch, Stellenbosch, South Africa. \\ ${ }^{3}$ Department of Mathematics and Applied Mathematics, Rhodes University, Grahamstown, South \\ Africa
}

(Communicated to MIIR on 31 October 2021)

Study Group: MISGSA2017, 16-27 January, 2017, Rhodes University, Grahamstown, South Africa.

Communicated by: Graeme Hocking

Industrial Partner: Rhodes University

Presenter: Viroshan Naicker

Team Members: C. P. Please, University of Oxford; R. Omer, AIMS, Cape Town and University of Stellenbosch; G. Kirsten, Rhodes University

Industrial Sector: Environment

Key Words: Dispersion, Pollution, Landfill

MSC2020 Codes: 76, 91

\section{Summary}

Data has been collected by residents in a neighbourhood of Durban concerning noxious odours and health issues. This report examines how this data might be used to identify possible sources of the reported smells and their level of emission. The approach is to exploit a well known simple mathematical model of dispersion of chemicals and use this to look at the inverse problem of identifying possible sources. Two of the complicating issues are that, unlike most previous work, i) the recorded data is subjective, indicating only that a smell was detected with no indication of concentration, and ii) there are large amounts of missing data, both due to no reporting of low concentrations and not always reporting high levels. This makes the inverse problem slightly non-standard.

$\dagger$ Corresponding author: colin.please@maths.ox.ac.uk 


\title{
POLLUTION FROM HAZARDOUS LANDFILL SITES
}

\author{
C.P. Please*, R. Omer ${ }^{\dagger}$ and G. Kirsten ${ }^{\ddagger}$ \\ Industry Representative
}

Viroshan Naicker ${ }^{\S}$

\begin{abstract}
Data has been collected by residents in a neighbourhood of Durban concerning noxious odours and health issues. This report examines how this data might be used to identify possible sources of the reported smells and their level of emission. The approach is to exploit a well known simple mathematical model of dispersion of chemicals and use this to look at the inverse problem of identifying possible sources. Two of the complicating issues are that, unlike most previous work, i) the recorded data is subjective, indicating only that a smell was detected with no indication of concentration, and ii) there are large amounts of missing data, both due to no reporting of low concentrations and not always reporting high levels. This makes the inverse problem slightly non-standard.
\end{abstract}

\section{Introduction}

There are a number of possible sources of hazardous pollution in the area around Durban and local residents have organised to collect data in an attempt to identify

*Oxford Centre for Collaborative Applied Mathematics, University of Oxford, Oxford OX1 3LB, United Kingdom.email: colin.please@maths.ox.ac.uk

${ }^{\dagger}$ African Institute for Mathematical Sciences, Muizenberg, Cape Town, South Africa. email: rene@aims.ac.za

${ }^{\ddagger}$ Department of Mathematical Sciences, University of Stellenbosch, Stellenbosch, South Africa. email:17578973@sun.ac.za

$\S$ Department of Mathematics and Applied Mathematics, Rhodes University, Grahamstown, South Africaeamil: v.naicker@ru.ac.za 
if any of these might be the source of noxious odours and the cause of various health issues. The region of interest is shown in Figure 1 indicating where complaints have been logged and some of the possible sources.

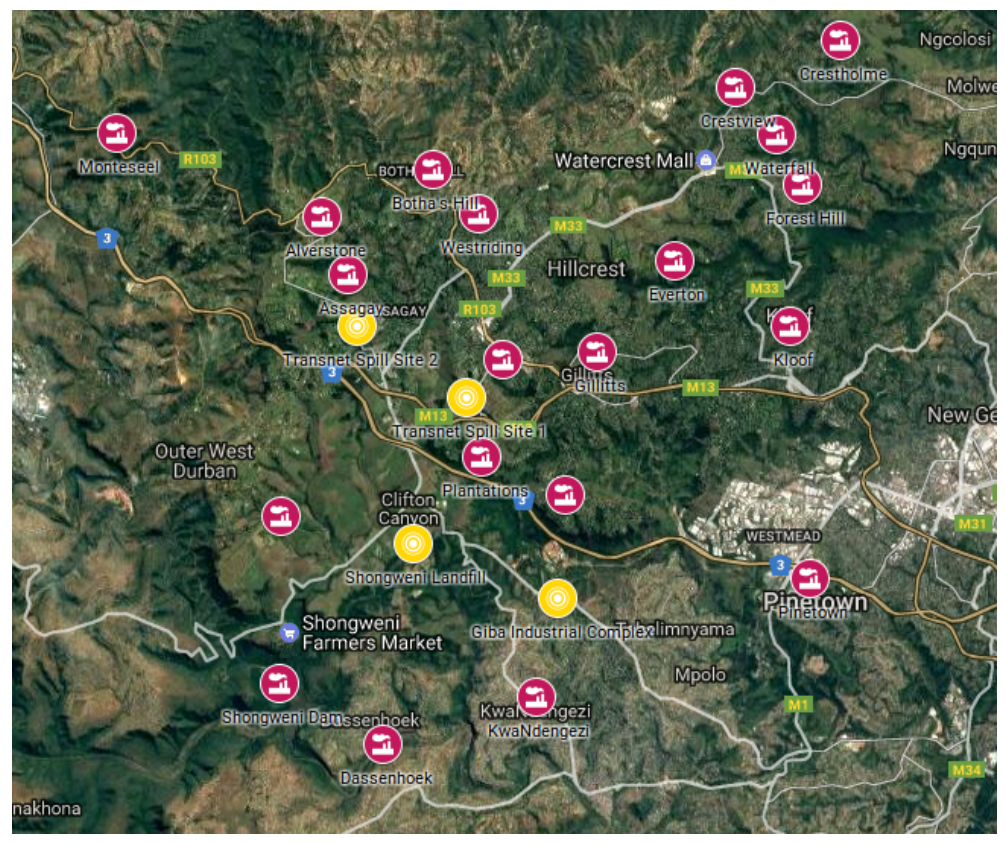

Figure 1: Plan view of region of interest. Red dots are approximate locations of complaints, Yellow dots are some possible sources of odours, blue lines are motorways. Produced using Google MyMaps.

Sites which store hazardous materials can represent a serious challenge to public health. As toxins accumulate at the site and as the site ages air and ground water pollution levels increase which, in turn, adversely affects the environment around the site and imposes a negative economic externality on the neighbouring communities. These costs include long term environmental degradation, chronic health management costs, clean up costs, loss of income for local businesses, and decline of property values.

The Study group was asked to consider two questions. Firstly, if it was possible to model the spread and accumulation of pollution through the environment? For example, landfills are responsible both for air pollutants and for ground water pollutants. How would pollutants from a landfill site distribute themselves into the area surrounding the landfill? Airborne pollutants are necessarily carried by wind and moisture, while waterways distribute and dilute toxins from pollution sites.

The second question asked was if it would be possible to estimate the economic costs of the pollution? These include, but are not limited to, the above mentioned public health and medical expenditures, decline in property prices, loss of income 
and viable agricultural land and environmental clean up costs?

The Study Group concentrated on the first of these questions using mathematical models to explain the behaviour and addressing the question of how the data collected by residents might be used to indicate where possible sources of pollution were. The second question was not discussed at length but useful references that set out clear methodology for assessing the costs, and particularly health costs, of pollution were identified [8].

\section{Model of pollution dispersion}

Dispersion of chemical species within the atmosphere is a well studied area with many mathematical models developed to determine the behaviour. These models account for numerous physical phenomena and have varying degrees of complexity. The more complex models require numerical solution to determine the concentration distribution of the chemical for a given set of sources. These models are used extensively to predict the expected concentration of chemicals due to a particular source. In addition these concentrations are then used to quantify the health risk and cost of any particular source. There are various examples of models being used to predict concentrations of pollutants from known sources such as [1] but a particularly good example of such a model is the Danish Eulerian Hemispheric Model outlined in [2] and based on material in [3]. The model presented here is one of the simple versions of these models which can give reasonable predictions of the concentration and requires only a few key parameters that can be estimated from weather data.

The model considered is called the "Gaussian Dispersion Model" and is a slightly idealised situation in which there is a single species of chemical emitted from a single point source (the linearity of the situation, where each species and source does not affect the others will allow multiple sources and multiple chemicals to be considered if required). Furthermore we shall assume the atmospheric conditions are sufficiently constant that a steady plume is formed. We also take the ground to be level and for the source of the chemical to be at the ground level. The mechanisms that transport the chemical will be taken to be advection by the wind and dispersion by the turbulence of the atmosphere. The wind will be assumed to be steady, in both direction and strength, and to be uniform both vertically and horizontally. We shall also assume that the dispersion is constant and uniform. Note that these are reasonable assumptions for the situation here so long as the data is interpreted for the time when the detection has occurred. The model will predict the concentration everywhere but we shall be interested in the concentration on the ground since that is where any detection occurs.

Consider a single chemical species emitted from a point source creating a distribution with concentration $c(x, y, z)$, where $x, y$ are position in the horizontal plane 
and $z$ is the vertical position. The model will assume that the ground is flat but it is usually accepted that in cases where the ground is uneven $z$ can be interpreted as the vertical distance from the ground (we shall not invoke Egan terrain correction). We shall use a rotated coordinate system $(r, s, z)$ aligned with $(x, y, z)$ so that the wind blows towards the positive $r$ direction and $s$ is the horizontal distance perpendicular to the wind direction. The wind is take to blow at speed $U$ and there is horizontal dispersion $K_{s}$ and vertical dispersion $K_{z}$. As is conventional we neglect any dispersion in the $r$ direction since the wind strength is typically much larger than dispersion when considering any reasonable distances downstream of the source. Hence the equation governing the motion of the chemical is

$$
U \frac{\partial c}{\partial r}=K_{s} \frac{\partial^{2} c}{\partial s^{2}}+K_{z} \frac{\partial^{2} c}{\partial z^{2}}
$$

We want to solve this equation in the region $r \geq 0,-\infty<s<\infty$ and $0 \leq z<\infty$. We anticipate that the ground does not absorb any of the chemical and hence

$$
\frac{\partial c}{\partial z}=0 \quad \text { on } z=0 .
$$

Finally the source emits at a steady rate $Q(\mathrm{~g} / \mathrm{sec})$ from the point $(0,0,0)$ and hence the boundary condition is

$$
c(0, s, z)=\frac{Q}{U} \delta(s) \delta(z)
$$

where $\delta$ is the Dirac delta function. (Note that care is needed in defining the Dirac delta function on the boundary of the region and the critical property is that the integral of the chemical flux near the source must be $Q$.)

This classical model of dispersion can be readily solved. This can be done in two ways. Firstly by noting that the concentration can be identified as the product of two one-dimensional dispersion problems, one in $(r, s)$ and the other in $(r, z)$, or secondly by nondimensionalising the problem. In the second case we take, assuming a typical distance from the source to the measurement point to be $L$, as scale

$$
r=L \bar{r}, \quad s=\sqrt{\frac{K_{s} L}{U}} \bar{s}, \quad z=\sqrt{\frac{K_{z} L}{U}} \bar{z}, \quad c=\frac{Q}{L \sqrt{K_{s} K_{z}}} \bar{c}
$$

The problem becomes

$$
\frac{\partial \bar{c}}{\partial \bar{r}}=\frac{\partial^{2} \bar{c}}{\partial \bar{s}^{2}}+\frac{\partial^{2} \bar{c}}{\partial \bar{z}^{2}}
$$

where,

$$
\frac{\partial \bar{c}}{\partial \bar{z}}=0 \quad \text { on } \bar{z}=0
$$

and

$$
\bar{c}(0, \bar{s}, \bar{z})=\delta(\bar{s}) \cdot \delta(\bar{z})
$$


(Note that we have used the property that $\delta$ has dimensions of the inverse of its argument.)

We can now either use separation of variables or note that the problem is radially symmetric to write the solution

$$
\bar{c}=\frac{2}{\pi \bar{r}} \exp \left(-\frac{\bar{z}^{2}+\bar{s}^{2}}{2 \bar{r}}\right)
$$

or in dimensional form

$$
c=\frac{2 Q}{\pi \sqrt{K_{s} K_{z}} r} \exp \left(-\frac{U\left(z^{2} / K_{z}+s^{2} / K_{s}\right)}{2 r}\right) .
$$

This completes the model for the concentration at a point for a single source.

\section{Analysis of data}

The data available for fitting the model and hence for investigating the source of the smells comes from complaints lodged by local residents using an online system. The data available to study has been collected from May 2016 through until the end of December 2016. Approximately 15,000 complaints have been logged in total. This data has been anonymised to allow it to be studied. The online system logs the time and date of the complaint and asks the resident for information related to their location (the anonymised data only indicates the residential community and not the address), the wind direction (this can be selected from eight points of the compass), the type of smell (a number of options are listed), and the health issues that have been occurring (a list is provided). There is also the option for the resident to input general text about the situation or to give more details or provide information not in the lists.

Using this data in its raw form has a number of issues that make analysis awkward. Firstly the sections where residents input text, such as the location, produce numerous spelling errors and incoherent answers. The wind direction may be inaccurate, although there is advice on the online system directing residents to where such data can be easily found. Some residents have not completed all the requested information such as numerous who give no type for the smell or any health issues. Many of these issues have been addressed by looking through the data and correcting spelling and removing complaints with inadequate information.

A particular issue is the assessment of the wind direction and the lack of more quantified data related to the weather conditions. Since the dispersion of chemicals is very dependent on these conditions an accurate independent source of the information could both validate the data that is given by residents and enhance the possibility of understanding where the smell originated from. Discussions with 
the South African Weather Service (SAWS) revealed that a weather testing station started continuous monitoring of the weather in the community of Hillcrest (see Figure 1 for approximate location) at the end of November 2016. It would therefore be extremely beneficial to use the data from this source to allow more accurate interpretation of the possible source of the smells. Using this data is an important future activity.

In order to fit the model it is necessary to estimate the wind conditions at the time that each complaint was made. In particular an estimate is needed of the wind direction so that the $(r, s, z)$ coordinates can be correctly rotated onto the spatially fixed $(x, y, z)$ coordinates. Furthermore an estimate of the average wind speed $U$ is needed. This direction and speed should be readily available from the weather data. The other parameters that need to be estimated are the dispersion coefficients $K_{s}$ and $K_{z}$. A suggested way forward is to identify the stability state of the air flow using information about the wind speed, incoming solar radiation and cloud cover. One possibility is to use a 6 point scale (A, B, C, D, E ,F) and the associated empirically-derived graphs as outlined on pages 53-56 of [4]. (Note, to translate between the two notations $\sigma_{x}=K_{s} / U, \sigma_{z}=K_{z} / U$.)

It is useful to give some summary of the existing data to indicate the amount of information available. As mentioned earlier the location of the complaints is indicated in Figure 1 showing the relative position to some possible sources. The number of complaints per community is shown in Figure 2 showing that there are certain areas where there are many more complaints than others. In Figure 3 the number of complaints in those areas with the highest number of complaints is shown and illustrates that the number of complaints has risen sharply over the period of May to December 2016. It is unknown whether the localisation of the complaints or the increasing number is due to increased levels of noxious odours or due to increased awareness by the residents.

\section{Methodology for fitting model to data}

The inverse problem of determining the source and strength of a chemical source from collected data is one of great interest to many practical situations. We note for example the methodologies outlined in [6] and [7] who use models similar to those presented here and consider sensor measurements that provide continuous data at prescribed positions, and the work of NOAA [5] who have used a complex 3-dimensional dispersion model to fit data from the Fukushima nuclear accident. The situation addressed in this report is slightly different to the conventional problem because of the form of the data. In particular the data does not give a time history of the concentration at a fixed point. Indeed the data only indicates that at a location the concentration exceeded a value determined by the resident. Further- 


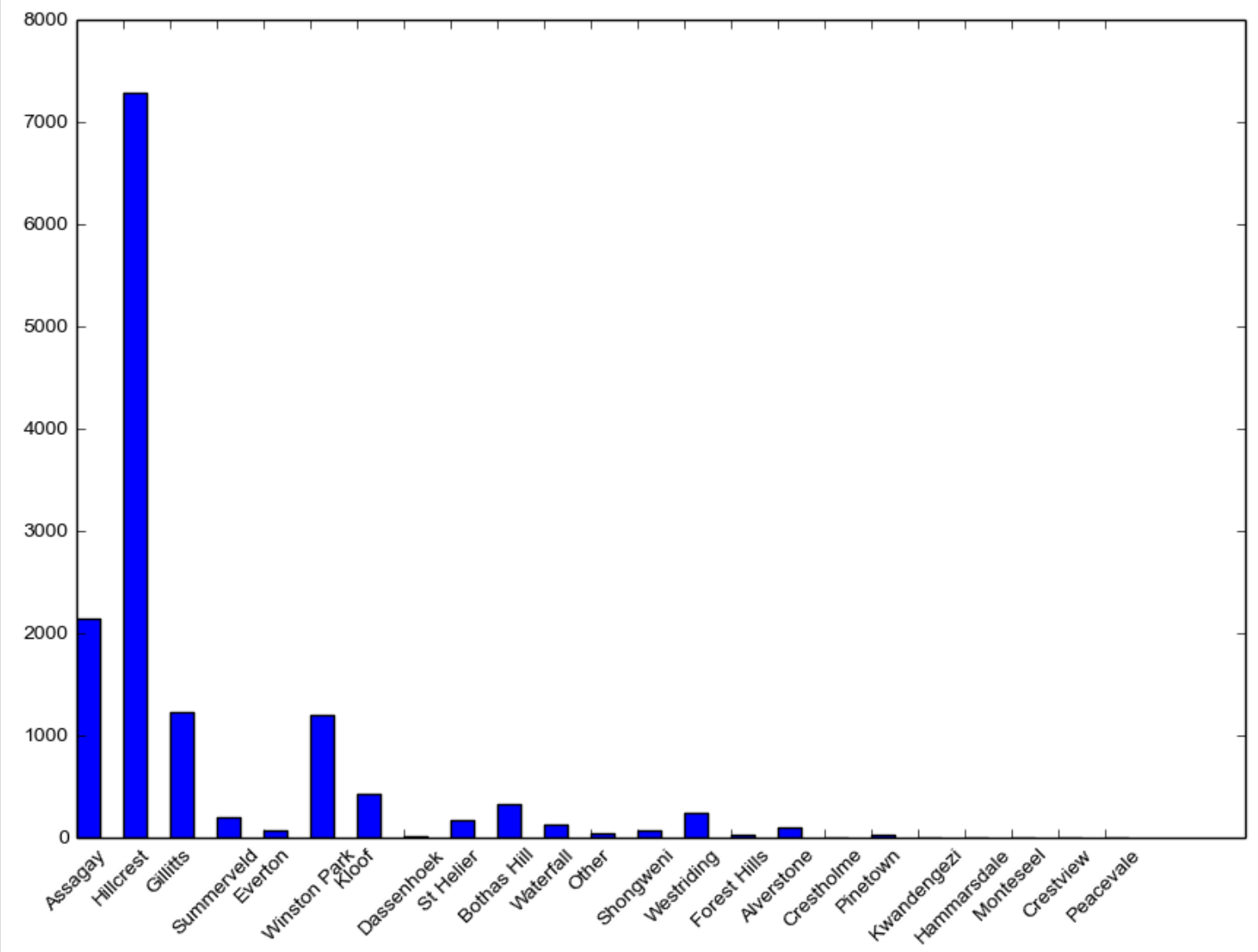

Figure 2: The number of complaints submitted from each community over the period May to December 2017 concerning noxious odours in the Durban area.

more we have no data on when the data was below that value at any point (residents have not submitted data on when they did not smell odours) and the data is incomplete in that not all residents submitted data every time the concentration exceeded their critical value. As a result it is not possible to use the methodologies outlined in much of the existing literature. Here we outline a methodology appropriate to understanding the consequences of the data as collected.

Our main tool of assessment is to use the collected data to indicate that at a location, during particular weather conditions a threshold concentration was exceeded. We do not have adequate knowledge to know what this threshold was. However, if we assume that there is only one source creating any one particular complaint event then the critical number is the ratio of the threshold values of the resident to the 


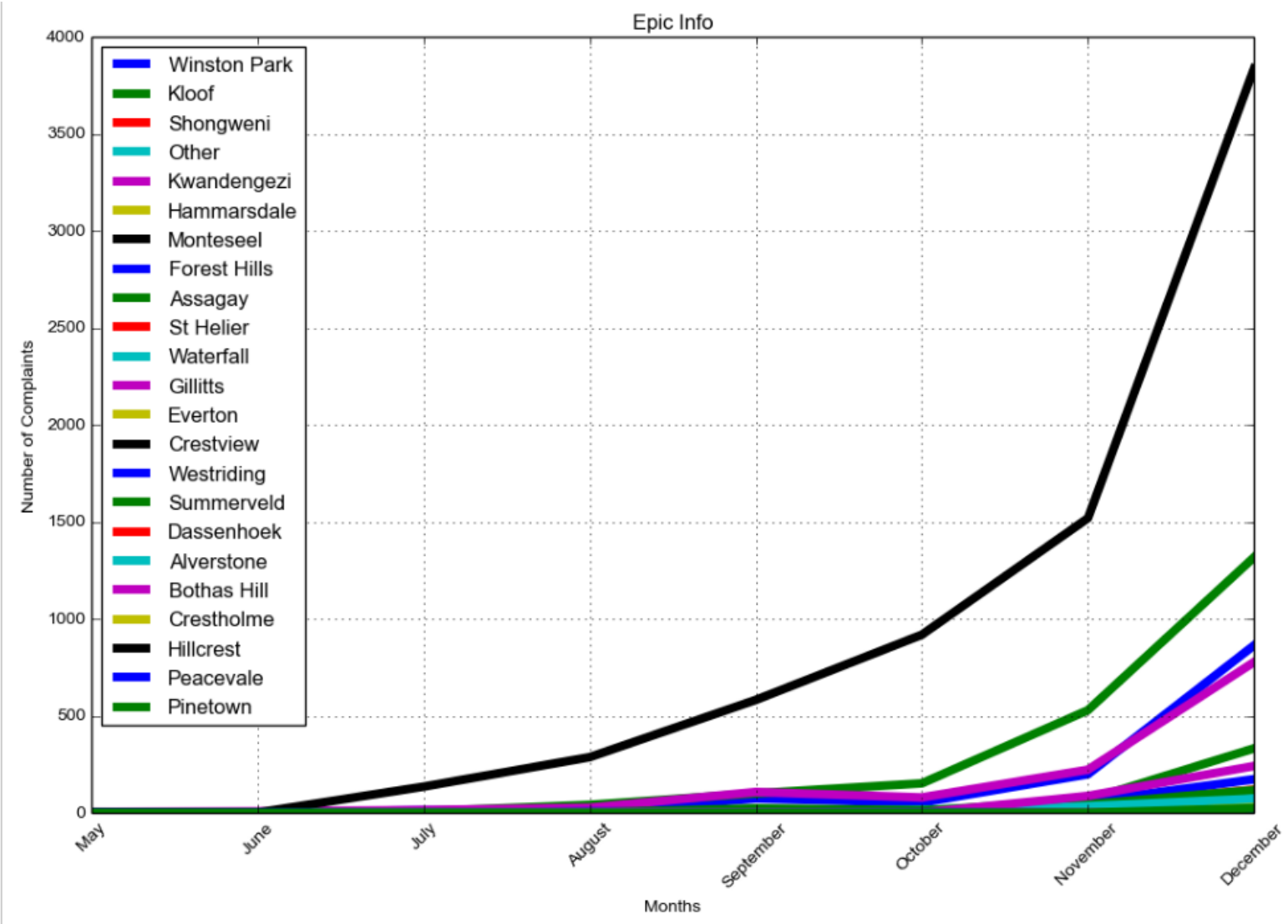

Figure 3: The number of complaints about noxious odours submitted each month from those communities with the largest number of complaints.

source strength. We call this ratio $\epsilon$ where

$$
\epsilon=c_{\text {crit }} \frac{u L^{2}}{Q}
$$

(where we shall choose $u=1 \mathrm{~m}^{2}$ and $L=1 \mathrm{~km}$ to make the units match but this makes no difference to the results). We will then explore where a source could be such that it gives a concentration greater than $c_{c r i t}$ which is related to $\epsilon$.

Our approach is to take each complaint and determine the region on the ground, we call this the "petal" of the complaint, in which the source must have occurred in order to make the concentration exceed a given value, related to $\epsilon$, at the complaint location. We can then assign a probability of there being a source for all positions in this petal. All complaints can then be assigned petals. By summing these probabilities and dividing by the total number of complaints we find the probability of a source being at any position for a particular value of $\epsilon$. This probability distribution will then be explored over a range of values of $\epsilon$ to determine possible source 
positions. Note this analysis does not preclude the possibility that there are several sources.

This approach will be good at identifying probabilities of sources but we need to include additional practical considerations. We note that one possible solution for high probabilities is that each complaint was due to a source very nearby, for example, burning toast in each kitchen, but we will assume that the odours are due to a relatively small number of sources. We also note that, since there is not data related to the absence of odours at any location that a single extremely large continuous source, where all residents experience large concentrations but only some complain occasionally, could explain the data. Again we exclude such a large source. We will therefore in fitting the data have to consider balancing probabilities of sources with the total number of sources and the maximum sources we anticipate in practice. This makes the problem a multi-objective optimisation problem.

The key step is to identify the petal of a complaint. This is readily done by solving for the possible positions for the source, relative to the complaint location, that give a concentration at the ground of $c_{\text {crit }}$. We use the same idea of rotated axes $(r, s, z)$ where $r$ points in the direction of the wind, but assume here that the origin $(0,0,0)$ is the site of the complaint. We assume that the source can be at position $(r=R, s=S)$, noting that the source will be upwind of the complaint so we expect $R<0$. The concentration is given, from earlier, by

$$
c_{c r i t}=\frac{2 Q}{\pi \sqrt{K_{s} K_{z}}(r-R)} \exp \left(-\frac{U\left(z^{2} / K_{z}+(s-S)^{2} / K_{s}\right)}{2(r-R)}\right)
$$

and hence the concentration is $\epsilon$ on the ground at the complaint location when

$$
\epsilon=\frac{2 u L^{2}}{\pi \sqrt{K_{s} K_{z}}} \frac{1}{(-R)} \exp \left(-\frac{U}{2 K_{s}} \frac{S^{2}}{(-R)}\right) .
$$

Rearranging this to identify the curve where the source might be gives the following petal shape

$$
(-\hat{R}) \ln (-\hat{R})=\hat{S}^{2}
$$

where, to allow us to see the generic shape of the petal we have defined

$$
R=\frac{u L^{2}}{\pi \epsilon \sqrt{K_{s} K_{z}}} \hat{R}, \quad S=L \sqrt{\frac{\pi u}{2 \epsilon U}}\left(\frac{K_{z}}{K_{s}}\right)^{1 / 4} \hat{S} .
$$

The petal shape is shown in Figure 4 where the complaint location is at $(0,0)$ and the curve surrounds the region where possible sources must occur in order for the concentration at the origin to be at the critical detection level. It is worth noting that the petal is of finite extent, because a more distant source will produce low concentrations. Similarly the petal is quite wide near the complaint site because diffusion will spread the chemical rapidly near the source. 


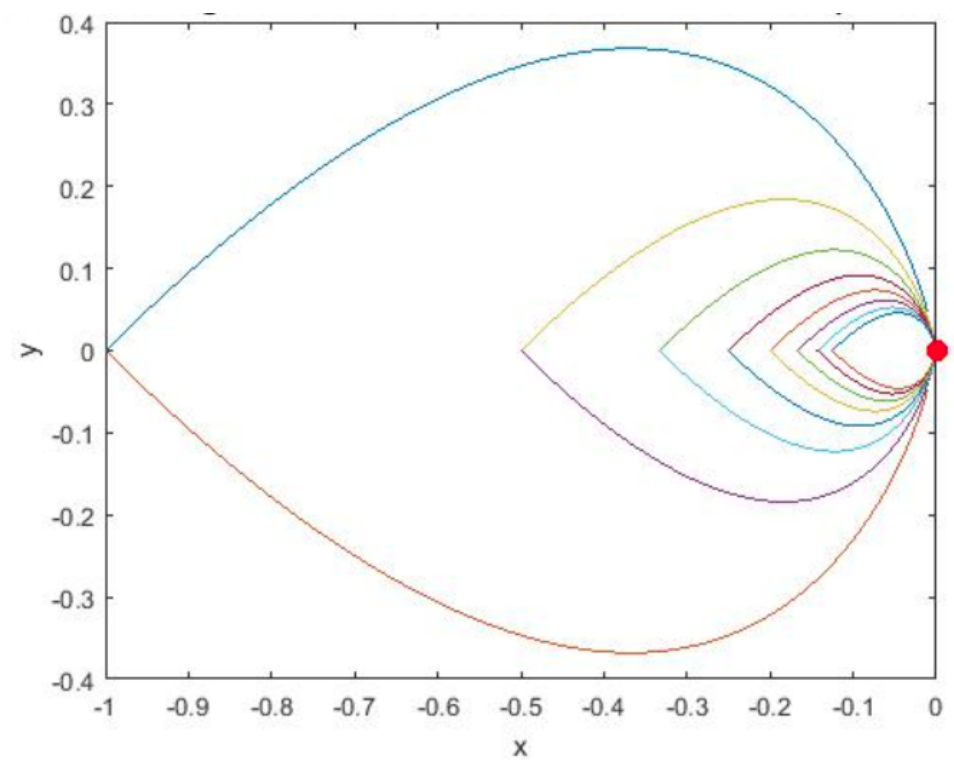

Figure 4: The "petal" for a single complaint, at a location indicated by the red dot, showing the boundary of the interior region where a source must occur for the concentration to exceed the critical level at the complaint location. Eight possible values of $\epsilon$ are displayed.

To use the petal we note that its area is given by

$$
A_{\text {petal }}=\frac{\pi L^{3}}{2} \sqrt{\frac{\epsilon u^{3}}{3 U}}\left(\frac{1}{K_{s}^{3} K_{z}}\right)^{1 / 4}
$$

(using $\int_{0}^{1} \sqrt{-x \ln x} d x=1 / 3 \sqrt{2 \pi / 3}$ ). Hence, since the probability of a source being in the petal is unity, and since there is no additional information, then it is reasonable to assume the probability is uniformly distributed in the region with probability per unit area inside the petal of

$$
P=\frac{2}{\pi L^{3}} \sqrt{\frac{3 U}{\epsilon u^{3}}}\left(K_{s}^{3} K_{z}\right)^{1 / 4}
$$

It now remains to compute the petal for each complaint for specified value of $\epsilon$ and use these collectively to determine the total probability, per unit area, of a source by adding the individual probabilities and dividing by the number of complaints. This total probability then needs to be explored for a variety of values of increasing $\epsilon$. We anticipate that for small $\epsilon$ the maximum probability will be around each complaint and this corresponds to the "many small sources" solution. As the size of $\epsilon$ increases we anticipate that new regions of maximum probability will appear as petals overlap, and in particular when petals from very different wind directions 
overlap to triangulate possible sources. If $\epsilon$ is further increased the probability everywhere will slowly decrease and become more uniform corresponding to the "single very large source" solution.

\section{Conclusions and future work}

A methodology has been outlined that allows the probability of a source being in a certain region to be determined as a function of one unknown parameter by exploiting a mathematical model of the physics of the problem and data from complaints and from the weather service. By varying the unknown parameter probable sources of the noxious odours should be identified in a systematic manner by excluding cases where the number of sources is very large or where a very few sources have an extremely large emission rate. This should provide a good way of understanding the complaint data in terms of possible sources.

Once probable pollution sources are identified it is necessary to assess the costs associated with such pollution. We have not done extensive work in this area but the work by [8] sets out a very clear methodology for assessing the cost implications of pollution sources. They use a dispersion model to compute expected concentration levels and then convert these to health implications allowing them to compute the expected health cost of any source of pollution. In these assessments they use complicated, detailed and computationally expensive models of the dispersion of the pollutants, because they exploit the fact that they know the strength of the sources, but the methods are equally applicable to the situations where a simpler model has been used to fit the existing data.

\section{References}

[1] Berkowicz, R., A simple model for urban backgound pollution, Environmental Monitoring and Assessment, 65, 2000, 259-267.

[2] Danish Eulerian Hemispheric Model (DEHM) (in Danish), 2017, envs.au.dk/en/knowledge/air/models/dehm/ .

[3] Christensen, J.H., The Danish Eulerian Hemispheric Model - A three dimensional air pollution model used in the Artic, Atmospheric Environment, 31, 24, 1997, 4169-4191.

[4] courses.washington.edu/cee490/PlumeD4.pdf

[5] www.arl.noaa.gov/Inverse_mdl.php , 2016, National Oceanic and Atmospheric Administration, US Department of Commerce 
[6] Dokmanić, I., Ranieri, J., Amina Chebira, A., and Vetterli, M., Sensor Networks for Diffusion Fields: Detection of Sources in Space and Time, $49^{\text {th }}$ Annual Allerton Conference, Illinois, USA, September 2011, pp 28-30.

[7] Nehorai, A., Detection and localization of vapour emitting sources, IEEE Trans. Signal Processing, 43 1, 1995, 243-253.

[8] "Cost Externalities of Air Pollution at the National Level using the EVA Model System", March 2011, www.ceeh.dk/CEEH_Reports/Report_3/ , ISSN 19047495 . 